\section{Pulmonary nodules: bringing order out of chaos}

\author{
Angela Morgan, ${ }^{1}$ Mark Slade ${ }^{2}$
}

Picture the scene: a lung cancer multidisciplinary team (MDT) meeting, somewhere in the UK, January 2015. CT images from a patient with suspected colorectal cancer, whom no one present has ever met, are discussed at the request of the colorectal team. An abnormality is present in the right lower lobe of lung. A week earlier, following two clicks and a drag from the colorectal radiologist's mouse, a $5 \mathrm{~mm}$ incidental pulmonary nodule was identified and is now destined to take its unwitting host on an unpredictable journey, which may span years. Imperfectly remembered advice from multiple professional society guidelines, some a decade old, will be proffered and contested in repeated meetings. How should the nodule be measured, how often, by whom? What is the risk that it is malignant? Is now (ever?) the right time for a biopsy? This scenario, which is likely familiar to many readers, has often served to relegate nodule follow-up to a tedious chore shared widely to ease the burden.

Help is at hand. With this issue of Thorax, David Baldwin, Mat Callister and colleagues publish the British Thoracic Society (BTS) guidelines for the investigation and management of pulmonary nodules, ${ }^{1}$ together with their own clear summary, ${ }^{2}$ which will support good, evidence-based patient care, effective resource use and audited outcomes. The precise and detailed distillation of a burgeoning evidence base-one-third of the 360 references are from 2012 or later-into four graphic algorithms represents a huge step from chaos towards order. It offers chest physicians everywhere the opportunity to channel the growing number of patients with incidental pulmonary nodules into a consistent and systematic process of investigation and, where appropriate, treatment.

A number of helpful simplifications emerge. Data from several large screening

${ }^{1}$ Department of Respiratory Medicine, Royal Wolverhampton Hospitals NHS Trust, Wolverhampton, UK; ${ }^{2}$ Department of Respiratory Medicine, Gloucestershire Hospitals NHS Foundation Trust, Cheltenham, UK

Correspondence to Dr Mark Slade, Department of Respiratory Medicine, Gloucestershire Hospitals NHS Foundation Trust, Cheltenham General Hospital, Sandford Road, Cheltenham GL53 7AN, UK; Mark.slade@nhs.net studies, including the Dutch NELSON study, ${ }^{3}$ provide clear evidence that nodules $<5 \mathrm{~mm}$ in diameter or $100 \mathrm{~mm}^{3}$ in volume have a very low risk of malignancy and do not require follow-up. The guideline authors have reduced the 'maximum ignorable volume' to $80 \mathrm{~mm}^{3}$ to allow for measurement discrepancies. It is made clear that the route by which nodules are identified, whether from screening studies, investigation of nonrespiratory symptoms or follow-up of a previously treated malignancy, should not affect their management. Nodules identified in a patient with lung cancer require careful assessment, given that they remain statistically more likely to be benign than metastatic. Although the confirmation of nodule features conferring a very low risk of malignancy is welcomed, we consider it likely that these will be confidently used only by specialist chest radiologists, making a report from this source highly valuable.

Elsewhere, things become a little more complex for the MDT. In come sub-solid nodules, a four-point scale for tracer uptake in reporting positron emission tomography (PET) scanning, and semiautomated volumetry. Out go semi-solid nodules and ground-glass opacities. Radiologists can throw away those callipers. We will all have to learn the new nodule lexicon.

Recent evidence confirms the value of semi-automated volumetric measurement of nodules. ${ }^{4}$ This makes estimates of volume doubling times more accurate and leads to better test performance in the prediction of malignancy by detecting malignant nodules in a much shorter time interval. Though they will be more timeconsuming for the radiologist to perform than simple calliper measurements, fewer nodules will require follow-up if volumetric assessments are performed at presentation, and where necessary follow-up will be briefer. Unfortunately, as would be expected, there are measurement discrepancies when different volumetric software packages calculate volumes for the same nodule. ${ }^{5}$ This variability will have to be accounted for in the management plan when nodules are followed up.

Published risk assessment tools perform better than expert opinion in assessing the risk of malignancy in pulmonary nodules, and the guideline recommendations reflect this. ${ }^{6}$ Where only clinical data and a CT scan result are available, the most accurate tool for predicting malignancy is the Brock University tool, ${ }^{7}$ which has an area under the receiver operator curve of 0.90 when validated in a UK population. PET-CT scanning is recommended if the risk of malignancy exceeds $10 \%$, when the Herder tool is employed. ${ }^{8}$ Although UK chest physicians are accustomed to frequent use of clinical risk assessment tools in the form, for example, of the CURB-65 score for pneumonia ${ }^{9}$ and the Wells score in suspected thromboembolism, ${ }^{10}$ each of these can be calculated in seconds by totting up a few integers. Calculating nodule malignancy risk will require a computer and a little more attention to detail. In our opinion, it is very important that doing so is not allowed to fall into the 'too-hard basket' in the course of the busy chest physician's or radiologist's day.

Readers hoping for a definitive answer to the frequently and hotly debated question, "Should we biopsy it first or should we just take it out?" will be disappointed. Although there is a very helpful, graphical illustration of the impact of a negative biopsy on the post-test probability of malignancy, and a careful examination of the impact of each strategy on length of stay, benign excision rates and hospital costs, we predict that preoperative biopsy will continue to be a glass half full to some and half empty to others. ${ }^{11}$ Respiratory radiologists who fear underemployment can console themselves that a booming practice in localisation techniques for pulmonary nodules, another recommendation, is just around the corner.

The guidelines represent a beautifully rigorous and clear summation of the current state of the evidence with respect to pulmonary nodules, but their recommendations are not simple, either to commit to memory or to implement accurately. In this respect, they exemplify the quotation, controversially attributed to Albert Einstein, that "Everything should be made as simple as possible, but not simpler". ${ }^{12}$ How should the MDT seek to implement the recommendations in order that their value to patients is fully realised? The authors propose that consideration be given to setting up dedicated pulmonary nodule services, staffed by individuals with sufficient commitment and expertise in this area to ensure that this is done. We wholeheartedly agree with this recommendation. In our opinion, several recommendations from 
the guidelines are unlikely to be successfully delivered outwith such a service, including those relating to

- consistent performance and reporting of serial follow-up imaging;

- the appropriate management of subsolid nodules;

- the confident exclusion of malignancy in nodules exhibiting clear features of intrapulmonary lymph nodes ${ }^{13}$ or hamartomas;

- appropriate application and interpretation of risk management tools;

- decision-making with respect to biopsy and treatment;

- the provision of accurate, appropriate patient information and support.

Concentrating the 'boring but important' work of nodule follow-up within a team of committed individuals is the best mitigation of the risk that a lung malignancy could be missed when at a resectable stage. Elsewhere, and primarily in procedural specialties, the risk that some element of a routine and possibly uninteresting process will be omitted, with potentially catastrophic consequences, has been very significantly reduced by the introduction of checklists, time-outs and 'stop the line' empowerment. ${ }^{14}$ Similar checklists could be built into the processes of a nodule service. It is likely that careful volumetric assessment of pulmonary nodules will be more deliverable if it is concentrated in the hands of a few radiologists in each hospital, where motivation will overcome the increase in time required to report each scan. This increase in reporting time is likely to be unpopular in radiology departments already under pressure. We therefore exhort those who run nodule clinics to evaluate the impact of the new guidelines on their patient cohorts in order to provide real-life experience concerning the balance between fewer follow-up scans versus longer reporting time.

Readers who take a more optimistic view of how readily the guideline recommendations can be implemented without such a service may like to consider how well the much simpler advice, to record the performance status and stage of all patients with lung cancer and submit them to the national lung cancer audit, was implemented in practice. The audit was in its eighth year of reporting, 2012, before the data completeness for either field achieved 90\%. ${ }^{15}$ What the experience of the national lung cancer audit has taught us, however, is the power of the National Health Service in collecting population-level data in lung cancer. The publication of the BTS guidelines for the investigation and management of pulmonary nodules offer us an opportunity to contribute a really influential, standardised and high-volume dataset to this important field. The guideline authors have consciously avoided being prescriptive in their discussion of how a nodule service might be run. We encourage them, after a well-deserved rest, to develop a standardised UK dataset for pulmonary nodule follow-up. If all clinicians are doing and measuring the same things, then there is the realistic hope that within a few years the data underpinning the management of incidentally discovered pulmonary nodules will be comparable to that derived from screening studies.

Competing interests None declared.

Provenance and peer review Commissioned; internally peer reviewed.

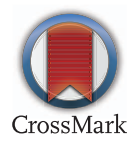

To cite Morgan A, Slade M. Thorax 2015;70:716717.

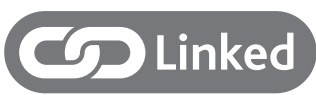

- http://dx.doi.org/10.1136/thoraxjnl-2015-207168

- http://dx.doi.org/10.1136/thoraxjnl-2015-207221

Thorax 2015;70:716-717.

doi:10.1136/thoraxjnl-2015-207466

\section{REFERENCES}

1 Callister MEJ, Baldwin DR, Akram AR, et al. British Thoracic Society guidelines for the investigation and management of pulmonary nodules. Thorax 2015; 70(Suppl 2):ii1-iii54.

2 Baldwin DR, Callister MEJ, On behalf of the members of the Guideline Development Group. The British Thoracic Society guidelines on the investigation and management of pulmonary nodules. Thorax 2015;70:794-8.

3 Horeweg N, van Rosmalen J, Heuvelmans MA, et al. Lung cancer probability in patients with CT-detected pulmonary nodules: a prespecified analysis of data from the NELSON trial of low-dose CT screening. Lancet Oncol 2014;15:1332-41.

4 Horeweg $\mathrm{N}$, van der Aalst $\mathrm{CM}$, Vliegenthart $\mathrm{R}$, et al. Volumetric computed tomography screening for lung cancer: three rounds of the NELSON trial. Eur Respir J 2013:42:1659-67.

5 Zhao YR, van Ooijen PM, Dorrius MD, et al. Comparison of three software systems for semi-automatic volumetry of pulmonary nodules on baseline and follow-up CT examinations. Acta Radiol 2014;55:691-8.

6 Swensen SJ, Silverstein MD, Edell ES, et al. Solitary pulmonary nodules: clinical prediction model versus physicians. Mayo Clin Proc 1999;74:319-29.

7 McWilliams A, Tammemagi MC, Mayo JR, et al. Probability of cancer in pulmonary nodules detected on first screening CT. N Engl J Med 2013;369:910-19.

8 Herder GJ, van Tinteren H, Golding RP, et al. Clinical prediction model to characterize pulmonary nodules: validation and added value of

18F-fluorodeoxyglucose positron emission tomography. Chest 2005;128:2490-6.

9 Lim WS, Woodhead M, British Thoracic S. British Thoracic Society adult community acquired pneumonia audit 2009/10. Thorax 2011;66:548-9.

10 Hogg K, Thomas D, Mackway-Jones K, et al. Diagnosing pulmonary embolism: a comparison of clinical probability scores. Br J Haematol 2011;153:253-8.

11 Fontaine-Delaruelle C, Souquet PJ, Gamondes D, et al. Negative predictive value of transthoracic core needle biopsy: a multicenter study. Chest. Published Online First: 8 Jan 2015. doi:10.1378/chest.14-1907

12 O'Toole G. Everything should be made as simple as possible, but not simpler. Quote Investigator [blog], 13 May 2011. http://quoteinvestigator.com/2011/05/ 13/einstein-simple/ (accessed 14 Jun 2015).

13 de Hoop B, van Ginneken B, Gietema H, et al. Pulmonary perifissural nodules on $C T$ scans: rapid growth is not a predictor of malignancy. Radiology 2012;265:611-16.

14 Haugen AS, Softeland E, Almeland SK, et al. Effect of the World Health Organization checklist on patient outcomes: a stepped wedge cluster randomized controlled trial. Ann Surg 2015;261:821-8.

15 Health and Social Care Information Centre. National Lung Cancer Audit 2014;p12 Fig 4. 